\title{
Embedding Ethical Impact Assessment in Nanosafety Decision Support
}

\author{
Ineke Malsch,* Panagiotis Isigonis, Maria Dusinska, and Evert A. Bouman
}

Nanotechnology is a key enabling technology, which is developing fast and influences many aspects of life. Nanomaterials are already included in a broad range of products and industrial sectors. Nanosafety issues are still a matter of concern for policy makers and stakeholders, but currently, there is no platform where all stakeholders can meet and discuss these issues. A comprehensive overview of all the issues in one single dashboard presenting the output of a decision support system is also lacking. This article outlines a strategy for developing one innovative part of a modular decision support system, designed to support the work of a new Risk Governance Council (RGC) for nanomaterials which will be established through the combined efforts of the GOV4NANO, NANORIGO, and RiskGONE H2020 projects. This new module will consist of guidelines for Ethical Impact Assessment (EIA) for nanomaterials and nanoenabled products. This article offers recommendations for adapting the European Committee for Standardization (CEN) prestandard on Ethical Impact Assessment CWA (CEN Workshop Agreement) 17145-2:2017 (E), to fit into the more-encompassing decision support system for risk governance of nanomaterials within the RiskGONE project.

\section{Introduction: RiskGONE at the Vanguard of Transparent Risk Governance}

The RiskGONE project and two other EU H2020 NMBP-13funded research projects (NANORIGO and GOV4NANO), targeting risk governance of nanomaterials, aim to resolve key

\section{Dr. I. Malsch}

Malsch TechnoValuation

PO Box 455, Utrecht, AL 3500, The Netherlands

E-mail: malschtechnovaluation@xs4all.nl

Dr. P. Isigonis

Department of Environmental Sciences

Informatics and Statistics

Ca' Foscari University of Venice

via Torino 155, Mestre-Venezia, Venice 30172, Italy

Dr. M. Dusinska

Environmental Chemistry Department

NILU

Instituttveien 18, Kjeller 2007, Norway

Dr. E. A. Bouman

Environmental Impacts and Sustainability

NILU

Instituttveien 18, Kjeller 2007, Norway

The ORCID identification number(s) for the author(s) of this article can be found under https://doi.org/10.1002/smll.202002901.

DOI: 10.1002/smll.202002901 issues in developing and curating scientifically sound data on nanomaterials and also concerning the role of stakeholder participation in risk governance, by establishing an RGC and an associated risk governance framework for nanomaterials. Complementary to the other two projects, RiskGONE puts considerable effort into the development and testing of a modular decision support tool incorporating risk assessment as well as consideration of economic, environmental, social, and ethical aspects from a holistic life cycle perspective. The RiskGONE approach is inspired by the International Risk Governance Council (IRGC) Risk Governance Framework and the Emerging Risk Management Framework at the core of the draft CWA 67 on "managing emerging technologyrelated risks." [1-3] A CWA is a prestandard, based on the work of a CEN Workshop of representatives of interested parties. After this workshop has agreed on the CWA, it remains valid for three years. At the end of this prestandardization period, the CWA can be extended for maximum another three years, revised, transformed into another deliverable, or withdrawn. ${ }^{[4]}$

This article focuses on the strategy for developing one new module in the larger decision support system for Risk Governance of nanomaterials. The new module consists of guidelines for EIA for nanomaterials and nanoenabled products. RiskGONE aims to adapt and test the procedures described in the CEN CWA 17145-2:2017 (E) part 2 on EIA for research and innovation, which was originally developed in the EU-funded project SATORI. ${ }^{[5,6]}$ This paper offers key recommendations with a view to adapting this prestandard, to fit into the more-encompassing decision support system under development within the project.

\section{The Science Policy Problems Addressed by RiskGONE}

Globally, the environmentally sound management of chemicals should have been achieved by 2020. ${ }^{[7]}$ While this is difficult enough for traditional chemicals, risk governance of nanomaterials is even more challenging, because the environmental, health, and safety risks are still not fully assessed. ${ }^{[8]}$ It is not clearly defined among policy makers, stakeholders, and key actors, neither what constitutes appropriate risk governance, 
nor which are the boundaries of regulatory compliance and legal basis of risk governance approaches. In this context, evidencebased (i.e., taking into account peer-reviewed, interdisciplinary scientific data and knowledge, including risk assessment, life cycle assessment and economic, social and ethical aspects) and participative (i.e., engaging all stakeholders in decision making) risk governance of nanomaterials is hampered by three interrelated conundrums.

1. Evidence-based scientific analysis is presently hampered by a lack of interoperability of reliable data from risk assessment studies in the public domain.

2. There is a lack of a one-stop-shop that could bring together all evaluated and curated data in one dashboard to facilitate decision making.

3. There is a pervasive lack of broad citizen and stakeholder engagement in decision making about nanotechnology.

The widely recognized lack of interoperability of curated data is expected to be (partially) resolved in the coming years. Several governments have developed roadmaps for nanoinformatics and omics, and the issue of creating consistent and reliable data using agreed upon methods is addressed by many research projects coordinated at the international level by the OECD WPMN testing programme, the ELIXIR portal ${ }^{[9]}$ in the European Union (working in cooperation with the USA National Institutes of Health) and a host of national governments. The lack of a one-stop-shop hampers rational decision making based on all available evidence while considering the quality of the data and the remaining uncertainties. This problem is addressed in numerous research projects developing Decision Support Systems for nanomaterials. ${ }^{[10-12]}$

The lack of stakeholder awareness remains highly problematic. Since the use of nanotechnology is ubiquitous around the world, there should be much more broad-based participation than currently experienced, in line with the rights to access to environmental information, to public participation in environmental decision making and to access to justice under the Aarhus Convention (1998). ${ }^{[13]}$ This is even more pressing, because of persistent disagreements between relevant stakeholder representatives, notably between industries manufacturing nanoenabled products on the one hand and nongovernmental organizations including environmental and consumer organizations on the other.

This lack of public awareness is addressed by several European and national dialogue and engagement projects and is the topic of OECD guidelines developed in the OECD Working Party on Nanotechnologies. ${ }^{[1-16]}$

All these projects generate improved capacity building concerning data and tools for evidence-based decision making on nanomaterials governance. However, it remains unclear at this point whether policy makers have the knowledge base required to implement the science-based tools in practice or whether further collaboration with scientists is needed. Herman Stamm of the European Commission wrote in Nature in 2011 about the then-urgent need for policy makers to develop an integrated approach to risk governance of nanomaterials, based on reliable data, accessible in one dashboard through modular decision support systems. ${ }^{[17]}$
As science progresses within clusters of expert committees, one part of the conundrum may soon be resolved as nanoinformatics are under development following agreed upon protocols and will be curated along prescribed rules. A more subtle and perhaps more important facet of this conundrum involves making sure that the voices of all stakeholders and citizens are heard in line with the Aarhus Convention. Consensus is required not only about the scientific methods but also the political goals of nanoinformatics and nanoenabled products in commerce before key components of any future regime for nanotechnology risk governance can be resolved.

\section{Methods: The EIA Prestandard}

In 2017, the CEN published a CEN CWA on Ethics assessment for research and innovation. The CWA on EIA consists of two parts. Part 1: Ethics Committee, "makes recommendations for the composition, role, functioning, and procedures of [an] ethics committee. ... Ethics committees include, but are not limited to, research ethics committees, institutional review boards, ethical review committees, ethics boards, and units consisting of one or more ethics officers." [5]

Part 2 of the CWA on Ethics Assessment ("EIA framework"), "provides researchers and organizations with guidance on EIA a comprehensive approach for ethically assessing the actual and potential mid- and long-term impacts of research and innovation on society. Researchers and ethics committees will find this information useful as it describes EIA at different stages of the ethical assessment. Part 2 is applicable to all researchers and innovators, regardless of the context they are working in or their research and innovation area."[5] While the RGC to be established at the end of the collaboration of the three projects (i.e., GOV4NANO, NANORIGO, and RiskGONE), is not an ethics committee in the strict sense, part of its remit is expected to be to address ethical issues related to nanomaterials and nanoenabled products. Most members of the Council will not be researchers or innovators, but it will be expected to take position on the ethical impacts of nanomaterial research and innovation. This necessitates the development of a nanospecific EIA to be incorporated in the larger decision support system providing the evidence-based information backbone of the Council.

In the CEN CWA 17145-2:2017, EIA is defined as the "process of judging the ethical impacts of research and innovation activities, outcomes, and technologies that incorporates both means for a contextual identification and evaluation of these ethical impacts and development of a set of guidelines or recommendations for remedial actions aiming at mitigating ethical risks and enhancing ethical benefits, typically in consultation with stakeholders."[5] This definition implies that the prestandard promotes a form of procedural ethics, prescribing the steps to investigate ethical impacts, while leaving the choice of substantial ethical principles and values over to the organizations performing the assessment.

The EIA follows a six-step approach, outlined in the CEN CWA 17145-2:2017,[5] as seen in Figure 1. 
The first step is a "threshold analysis" to determine whether serious ethical concerns are involved. A self-assessment questionnaire listing general ethical concerns and scoring them on a Likert scale, from minor to severe, is recommended, and a check by an independent expert is required if no serious concerns are identified. If serious ethical concerns are identified, the second step is the preparation of an "EIA plan," determining the scope and budget of the EIA (small, medium, large scale project, or at the level of a technological field). The third step is the identification of ethical impacts, in consultation with stakeholders where feasible. Such consultations can be held online, but especially in large scale EIAs, face-to-face meetings are preferred, because this allows genuine dialogue and exchange of arguments. In this step, conceptual as well as empirical analysis methods can be used. The CEN CWA 17145-2:2017 includes an overview of methods that can be used in the ethical impact conceptual analysis, including ethical issues checklists and ethical theories for identifying explicit moral issues as well as intuitive methods. The empirical analysis makes use of expert solicitations. In the fourth step, the ethical impacts are evaluated, again preferably in consultation with stakeholders. The fifth step is the formulation and implementation of "remedial actions," and in the final sixth step, the EIA is reviewed and audited. The remedial actions can include compliance with ethical norms fitting in a variety of ethical theories. The CEN CWA 17145-2:2017 focuses mainly on laying out a procedure for performing an EIA and does not presuppose any specific substantial ethical theories.
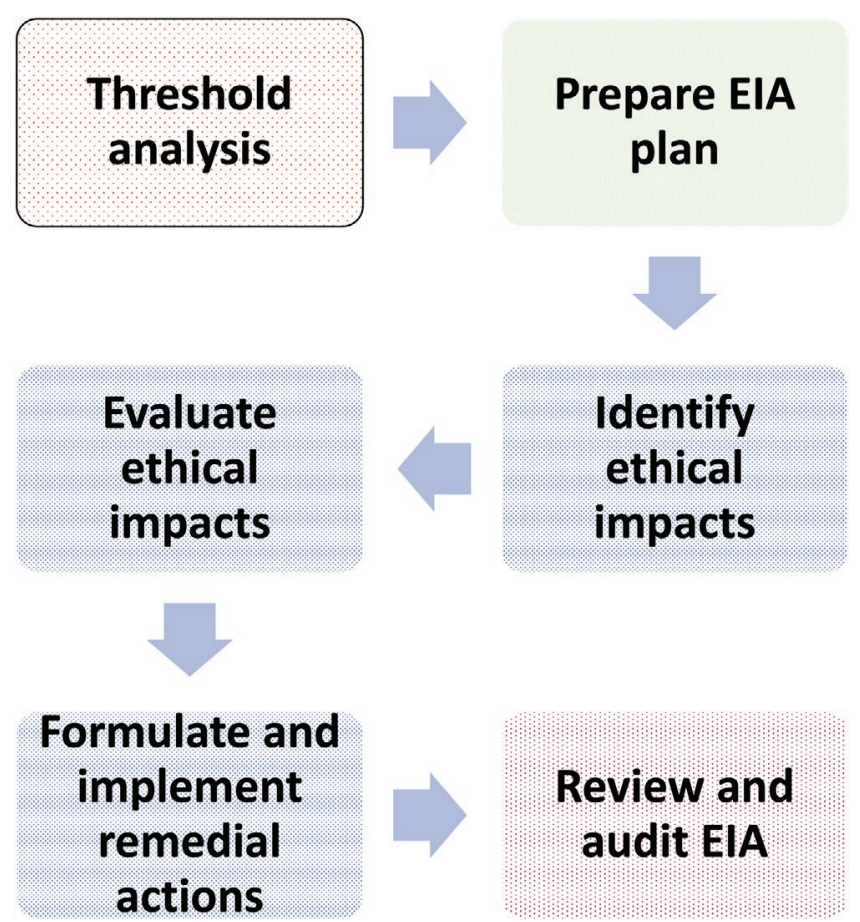

Figure 1. Illustration of the six-step EIA planning cycle, after CEN CWA 17145-2:2017 E. Adapted with permission from the original source.

\section{Including Ethics in Risk Governance}

To fit this procedure into the RiskGONE decision support system, it should be developed further into a decision-tree format, which can subsequently be implemented as a software-programme. The stepwise organization of the CEN CWA 17145-2:2017 offers a good starting point for such a decisiontree. In addition, overlap between the EIA and other modules of the decision support system should be omitted, to avoid double counting of the same issue. Notably, environmental, health, and safety issues, sustainability, risk management and transfer, and public opinion surveys are already addressed in other modules. The planned RGC is already expected to enable and encourage stakeholder consultations. This will limit the scope and resource requirement of the specific EIA module for nanomaterials, compared to the broader remit of the CEN CWA 17145-2:2017 E cited in Section 2. ${ }^{[5]}$ The EIA module under development in RiskGONE will focus on the identification of ethical risk thresholds for the risk-benefit assessment of nanomaterials. In addition, it should be tailored to serving the needs of the RGC which will be set up after the project. While the aims and scope of this Council will be defined in consultation with its prospective members, in general it should provide expert opinion on the governance of nanomaterials, not on market acceptance of an individual nanomaterial. The expected scope of the work of the RGC is therefore between a large-scale project-based EIA and an EIA for a whole new technological field (e.g., nanotechnology as an emerging technology, cf., CEN CWA 17145-2:2017 E, Annex B). ${ }^{[5]}$

The work of the RGC for nanomaterials will be modeled on the four-stage IRGC Risk Governance Framework. The EIA procedure fits seamlessly into this model because it consists of a similar cycle of preassessment, appraisal, evaluation, and management. The EIA threshold analysis and development of an EIA plan can be included in the risk preassessment. The risk appraisal stage can incorporate the identification of ethical impacts. The evaluation of ethical impacts fits in the risk characterization and evaluation stage. Finally, the risk management stage can cover the formulation and implementation of "remedial actions" for ethical impacts, and review and audit of the EIA (Figure 2).

\section{Selecting Relevant Ethical Theories and Concepts for Threshold Analysis}

Based on the identified ethical issues, thresholds identified for acceptability, tolerability, and intolerability of risks will be applied in selected nanotechnology sectors, to test the guidelines developed. If ethical impacts are deemed acceptable, no further action is needed, while if they are deemed tolerable, remedial actions should be undertaken, and if they are intolerable, the nanomaterials should be banned (see Figure 3).

For the purpose of this article, intolerable ethical issues include violating fundamental human rights principles such as the right to life and the prohibition of torture. The threshold between acceptable and tolerable ethical issues is more open to stakeholder deliberations and will be strongly influenced by 


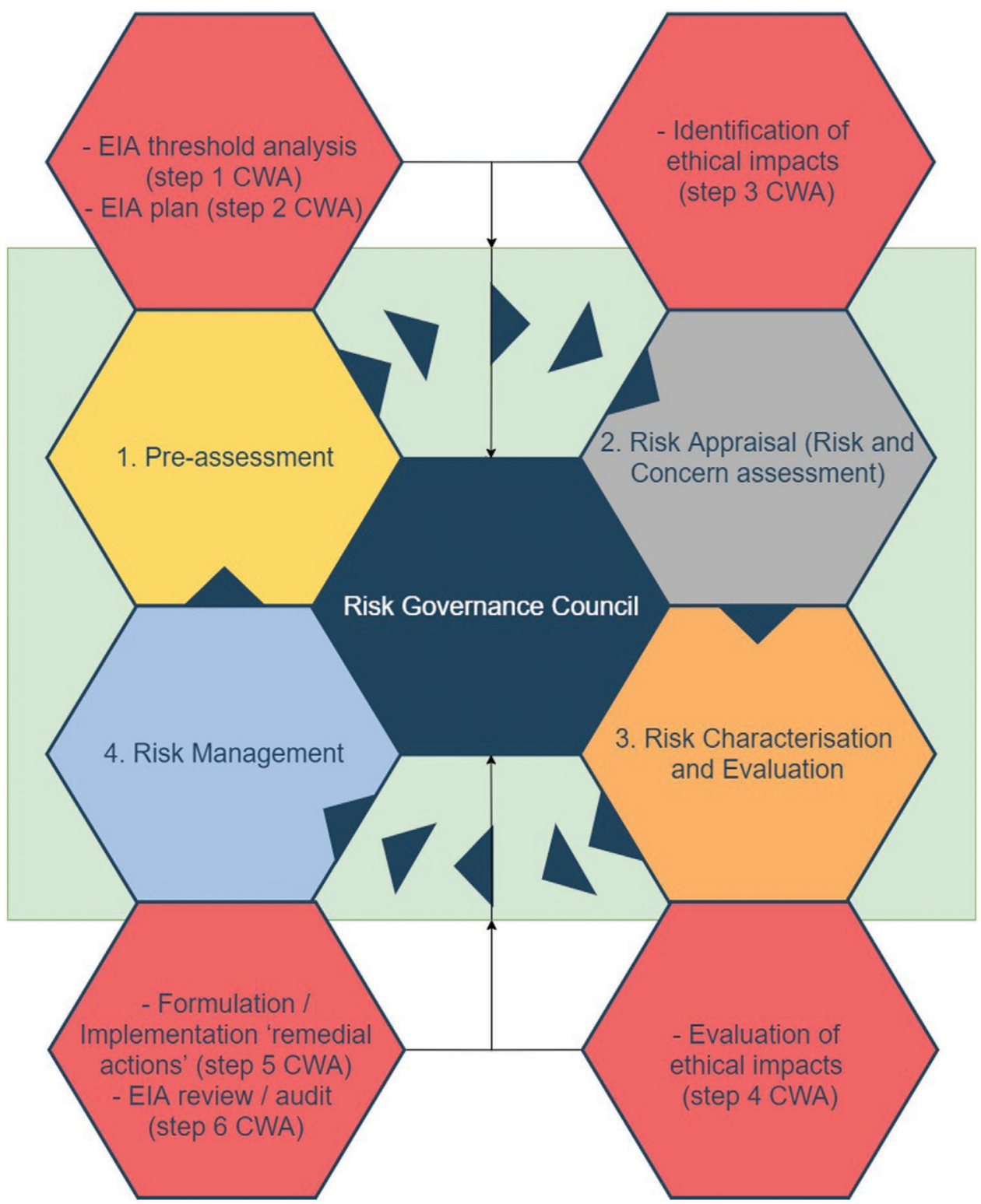

Figure 2. Incorporating the EIA in the Risk Governance framework.

the value systems of individual stakeholders. The intermediary domain between acceptable and intolerable risks is subject to the precautionary principle, but currently, there is no global

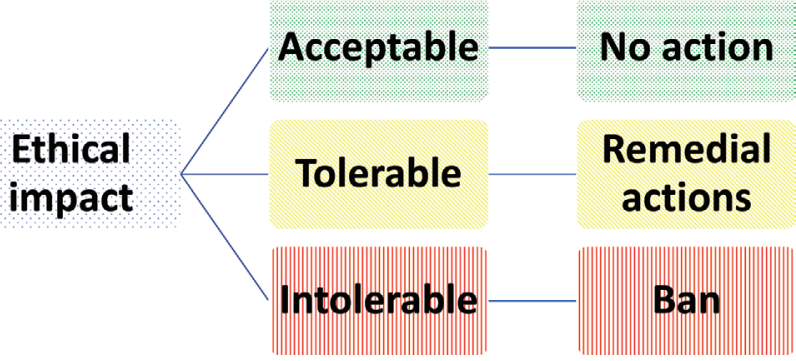

Figure 3. Decision tree sketching categories of ethical concerns and suggested actions. consensus on the definition of this principle. ${ }^{[18-20]}$ Meanwhile, the EU is incorporating a novel principle of "innovation" in the Horizon Europe regulation. ${ }^{[21]}$ Critics have questioned the ethical foundations of this principle, proposed by an industrial lobby group as a complementary concept to the precautionary principle, which is a founding principle in the treaty of the European Union. ${ }^{[2]}$ Whether these two goals are in opposition is currently a matter of debate.

The decision support tool can be used in identifying ethical impacts in a structured way, but the evaluation whether the identified ethical impacts are acceptable, tolerable, or intolerable requires stakeholder consultation.

As mentioned earlier, the CWA 17145-2:2017 E on EIA includes an overview of methods that can be used in the Ethical Impact Conceptual Analysis. These include mainstream ethical theories (deontology, consequentialism, and virtue ethics), 


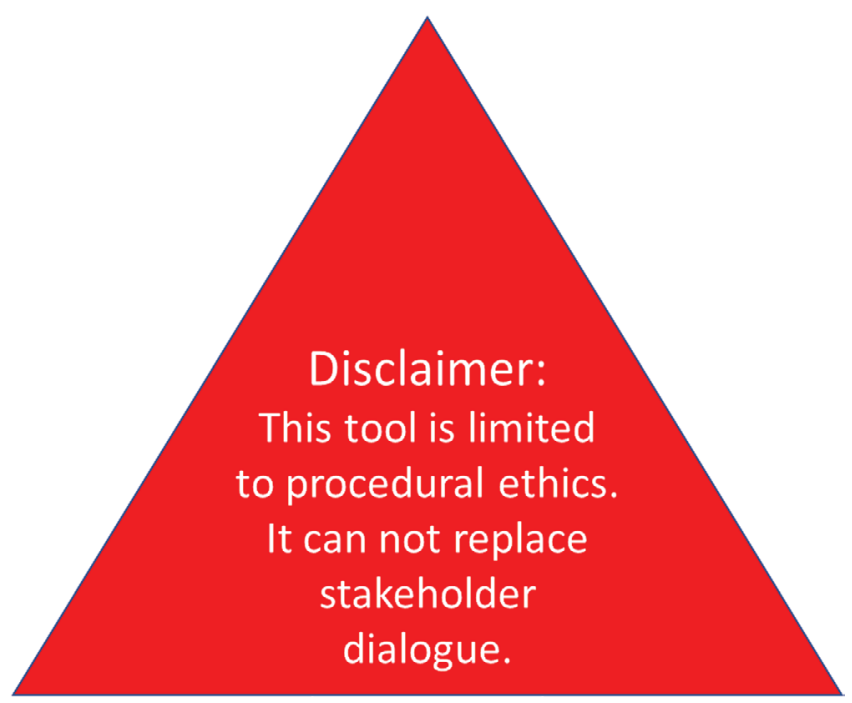

Figure 4. Disclaimer highlighting the limitations of the EIA module.

while other approaches, including care ethics and values, might also be used. However, not all these theories are compatible with a modular decision support tool. Deontology, calling for compliance with predetermined rules, and consequentialism, exploring the acceptability of expected future effects, are more likely to fit than deliberations about values, virtues, and care ethics. It is important to include a disclaimer warning the user of the proposed ethics module about these intrinsic limitations of the tool, as depicted in Figure 4.

As stated before, the EIA module will focus on the identification of ethical risk thresholds for the risk-benefit assessment (RBA) of nanomaterials. RBA can be considered inherently consequential as a positive or negative assessment is achieved by weighing potential risks (including costs) against benefits. Note that the risks, costs, and benefits are not necessarily of the same nature (e.g., monetary) and may affect agents differently across social, economic, and generational boundaries. This implies that the multicriteria valuation in RBA will necessarily reflect certain values. If only from a practical point of view, if the EIA is to inform other RBA modules (social, economic, environmental) it should at least reflect social (e.g., equity, gender), economic, and environment related values. To this extent, the user of the EIA module in the decision support tool, i.e., primarily the prospective RGC should consider among others the following theories and core values: human rights and human dignity, environmental ethics, ethics of dual use technologies, biomedical ethics, theory of justice and capability approach.

\section{What Remains Out of Scope}

Not all ethical traditions or theories fit into a natural scientific model underlying the Risk Governance framework. The decision-tree format of the proposed EIA module is unavoidably limited to procedural ethics, used to determine the seriousness of identified ethical issues, as informed by the relevant ethical theories and concepts listed above.
This includes compliance with rules, and determination of the present or expected future applicability of known ethical issues found in literature and through stakeholder and expert consultation.

Reflecting on ethical dilemmas cannot easily be accommodated in such a model. For example, what constitutes an appropriate balance between precaution and innovation? It depends on who you ask: NGOs and industrialists are not likely to agree on this any time soon. Cultural differences among different world regions make it difficult to reach consensus on common definitions of the terminology. Most ethicists and philosophers will not try to solve the dilemmas but delve into the underlying conflicting values and worldviews and place them in historical contexts. The resulting nuanced ethical analyses are hardly compatible with color-coded categories (green $=$ acceptable, yellow $=$ tolerable, and red $=$ intolerable) of the decision support system. Black boxing such extensive ethical analyses into these categories will raise more controversy than it solves, because the different stakeholders will question whose preferences prevail in the seemingly simple output of the EIA.

\section{Conclusions and Further Work}

The EIA prestandard is compatible with the Risk Governance Framework. Ethical aspects, principles, and theories can be incorporated as a procedural ethics module in a more encompassing decision support tool. The methodology is not yet robust. In the next steps, the feasibility of expanding the scope of the ethics module from risk to risk-benefit assessment will be investigated. The first stage will focus on nanomaterials but depending on the mandate of the RGC, this may be broadened in as yet undefined ways. Inspired by comments from the reviewers, we will also delve into an indepth analysis of relevant ethical theories and concepts, as well as reviewing nanoethics, shedding light on the desirability of the attempt to incorporate ethics into a natural scientific decision support framework for risk governance of nanomaterials.

\section{Acknowledgements}

The comments and suggestions of two anonymous reviewers are gratefully acknowledged. This research received support from the European Commission H2020 project RiskGONE (Grant No. 814425).

\section{Conflict of Interest}

The authors declare no conflict of interest.

\section{Keywords}

ethics, guidelines, nanomaterials, nanosafety, regulation

Received: May 8, 2020 Published online: 
[1] O. Renn, P. Graham, Risk governance: Towards an integrative approach, IRGC, Geneva 2006.

[2] IRGC, Introduction to the IRGC Risk Governance Framework, revised version, https://doi.org/10.5075/EPFL-IRGC-233739 (accessed: June 2020).

[3] CEN Workshop Agreement (CWA), Managing emerging technologyrelated risks, Report no. CWA 16649, 2013.

[4] CEN Workshop Agreement (CWA), https://boss.cen.eu/developingdeliverables/CWA/Pages/default.aspx (accessed: June 2020).

[5] CEN, CEN Workshop Agreement CWA 17145-2:2017-Ethics assessment for research and innovation-Part 2: Ethical impact assessment framework, CEN, Brussels 2017.

[6] SATORI FP7 Project, http://satoriproject.eu (accessed: June 2020).

[7] UN, Report of the World Summit on Sustainable Development, UN, Johannesburg, South Africa 2002.

[8] P. Isigonis, D. Hristozov, C. Benighaus, E. Giubilato, K. Grieger, L. Pizzol, E. Semenzin, I. Linkov, A. Zabeo, A. Marcomini, Nanomaterials 2019, 9, 696.

[9] Elixir Platform, https://elixir-europe.org/(accessed: June 2020).

[10] I. Malsch, V. Subramanian, E. Semenzin, D. Hristozov, A. Marcomini, Environ. Syst. Decis. 2015, 35, 54.

[11] I. Malsch, V. Subramanian, E. Semenzin, A. Zabeo, D. Hristozov, M. Mullins, F. Murphy, I. Linkov, A. Marcomini, Environ. Syst. Decis. 2017, 37, 465.
[12] S. N. Sørensen, A. Baun, M. Burkard, M. Dal Maso, S. Foss Hansen, S. Harrison, R. Hjorth, S. Lofts, M. Matzke, B. Nowack, W. Peijnenburg, M. Poikkimäki, J. T. K. Quik, K. Schirmer, A. Verschoor, H. Wigger, D. J. Spurgeon, Environ. Sci.: Nano 2019, 6, 505.

[13] UNECE and EC, Aarhus convention, https://ec.europa.eu/environment/aarhus/ (accessed: June 2020).

[14] I. Malsch, V. Subramanian, E. Semenzin, D. Hristozov, A. Marcomini, M. Mullins, K. Hester, E. McAlea, F. Murphy, S. A. M. Tofail, J. Nanopart. Res. 2015, 17, 215.

[15] OECD, Planning guide for public engagement and outreach in nanotechnology, OECD, Paris 2012

[16] I. L. Feitshans, Global Health Impacts of Nanotechnology Law: A Tool for Stakeholder Engagement, Vol. 1, Jenny Stanford Publishing, New York, NY 2018

[17] H. Stamm, Nature 2011, 476, 399.

[18] COMEST, The precautionary principle, UNESCO, Paris 2005.

[19] EU, TREATY ESTABLISHING THE EUROPEAN COMMUNITY TITLE XVI - ENVIRONMENT - ARTICLE 130R, EU, Brussels 1992.

[20] Wingspread Statement on the Precautionary Principle, Wingspread Conference Center, WI, USA 1998.

[21] R. Madelin, Opportunity Now: Europe's Mission to Innovate, EU, Luxembourg 2016.

[22] K. Garnett, G. Van Calster, L. Reins, Law, Innovation Technol. 2018, 10, 1. 